\title{
Construction Treatment Technology for Cracks in Asphalt Pavement of Road and Bridge
}

\author{
Yueshu $\mathrm{Li}^{1}$ \\ ${ }^{1}$ Jilin Transportation Vocational Technical College
}

\begin{abstract}
In recent years, with the development of road traffic in China, road and bridge projects have gradually increased. While the number of projects has increased sharply, construction technology has been greatly improved. The increase in the use of roads and bridges has led to an increase in the probability of cracks on the roads and bridges. Starting from the common types of cracks, this article explains how to effectively ensure the service life of roads and bridges, improve the performance of roads and bridges, avoid road cracks, deal with the cracks, and emphasize the influencing factors of cracks and the corresponding solutions for the reference of relevant personnel.
\end{abstract}

\section{COMMON TYPES OF CRACKS}

\subsection{Fatigue cracks}

Fatigue cracks are a common form of cracks on roads and bridges (see figure 1). Every road and bridge has strength and longevity. Some roads and bridges bear the load imposed by the vehicle for a long time, so the stress is much greater than the strength, and the structural resistance is reduced. At this time, cracks and even fatigue fractures will occur on the road surface[1].

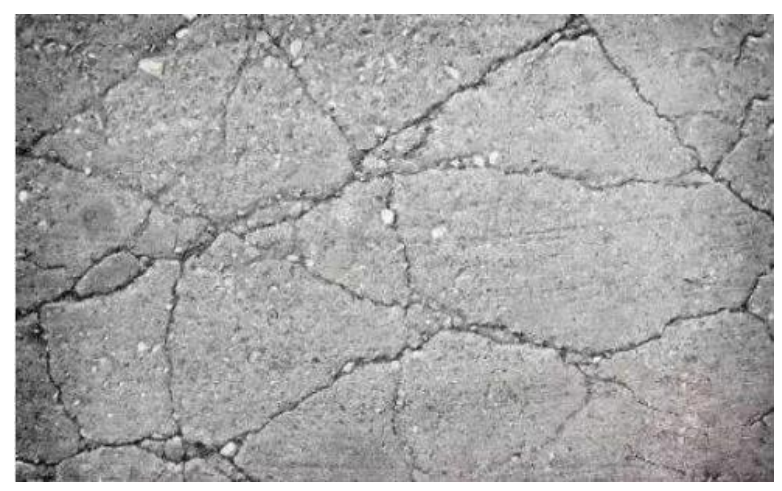

Figure 1. fatigue cracks

\subsection{Reflection cracks}

The reinforcement of semi-rigid substrate takes longer. If the quality of the construction can not meet the standard, it will be easy to cause temperature fatigue cracks and cold shrinkage cracks when the external temperature and humidity change. Tensile stress is generated due to the wear resistance of the roadbed. Once the tensile stress continues to increase and it is greater than the tensile strength of the foundation, the foundation will crack and generate force. Under load and temperature stress, the stress is concentrated on the weak points, causing the surface reflection cracks.

\subsection{Temperature cracks}

The thermal conductivity of each structural layer material of asphalt road surface is different, and the material itself is also affected by temperature. In the permeable structure of the asphalt road surface layer, it takes a certain time for the temperature to transfer, which will cause a large change in each layer of the structure. And it will show a clear temperature gradient especially when the ambient temperature is repeated[2]. The temperature gradient mainly causes corresponding stresses in the temperature layers of different structures, including cold shrinkage cracks and temperature fatigue cracks. The shape of cold shrinkage cracks is clearer mainly in high temperature environments, because asphalt is more flexible and deformed more obviously. Although the effect of temperature does not cause greater stress, in a cold environment, asphalt has obvious shrinkage under the condition of gradual hardening. Due to frequent temperature changes, especially in the case of large temperature differences between day and night, temperature fatigue cracks have a certain effect on the asphalt surface.

\section{INFLUENCING FACTORS OF ASPHALT CRACKS OF ROAD AND BRIDGE}

\subsection{The bearing capacity of road and bridge construction}

After the construction of roads and bridges is completed, it enters the use stage. Vehicle driving will affect the performance of the entire highway, and changes in the internal structure are likely to cause road diseases. When 
designing asphalt road surface, it is necessary to combine the future road and bridge uses with the number of vehicles and vehicle types to conduct a comprehensive analysis, study the unloading of vehicles and try to perform external control. In this way, the impact of the load on the road surface can be reduced and the efficiency of the road surface can be improved[3].

\subsection{Temperature changes}

As the temperature changes, the asphalt surface of roads and bridges will also be affected by temperature, causing thermal expansion and cold contraction. Asphalt substances will be affected by temperature changes, so the structure will change, and the original structure will also change to varying degrees. Therefore, it is necessary to analyze the resistance barrier[4]. If the temperature change exceeds the material's bearing capacity, cracks will appear, which will affect the driving of the vehicle.

\subsection{Smoothness of the surface construction of road and bridge}

In the construction of road and bridge projects in our country, it is necessary to ensure the construction quality of the project and reduce the engineering diseases in order to ensure people's travel safety. It is necessary for the relevant departments to improve the smoothness of the asphalt surface construction. If the road surface is not constructed in strict accordance with the specifications, the uneven road surface will affect the driving speed, increase the driving risk, and reduce the safety of the road construction. Therefore, it is imperative to improve the safety of the road surface.

\subsection{Factors of asphalt road surface materials}

For road and bridge engineering, the use of asphalt mixture is the basis of road construction. Generally speaking, the materials of asphalt road are mainly asphalt and mineral materials and the asphalt quality must meet the requirements of engineering standards. However, in actual construction, due to the tight schedule of the construction company, the arduous task, and the lack of professional analysis and testing during procurement, serious problems occur in the quality of some asphalt materials. Many asphalt materials have high wax content, resulting in poor elongation and low sensitivity. In the construction process, this material cannot produce strong adhesion, which leads to the inability to respond to temperature changes and the cracks occur. In addition, the use of mineral materials will also affect the quality of the asphalt mixture. If its technical parameters cannot meet the specifications, it will cause early cracking of the asphalt road surface.

\subsection{Improper use of the paver}

In the process of laying asphalt, the use of mechanical equipment is very common. The use and operation of mechanical equipment will also affect the construction quality of the project. Paver is one of the most important equipment on the highway, which has a direct impact on the overall structure of the highway. In addition, the stop running of the paver, the accumulation of the road causing by the pause of road construction, or the emergency stop will cause the problem of incomplete compaction of the road. Over time, cracks will appear in the indentation.

\section{TREATMENT TECHNOLOGY FOR CRACKS IN ASPHALT PAVEMENT OF ROADS AND BRIDGES}

\subsection{Scientific transportation of construction materials}

Asphalt materials are very common in road and bridge construction. Therefore, it is necessary to strictly control the use of asphalt raw materials during the construction process and conduct strict random inspections on the materials to ensure that the quality of the materials meets the requirements of the specification before they are used for engineering construction. When transporting materials, a larger dump truck should be used to ensure the safety of material transportation. During transportation, it must be ensured that it is not affected by rain and other factors. The transportation vehicle must be cleaned before transportation. It is strictly forbidden to accumulate debris in the dump truck to affect the materials. After the transport vehicles are delivered to the construction site, professional and technical personnel are responsible for handling the vehicles to avoid accidents.

\subsection{Dealing with the joints of road and bridge asphalt pavement}

Before paving asphalt materials for road and bridge engineering, longitudinal joints should be treated. During the construction process, if the road surface needs to be widened, the joints should be treated and arranged according to the paving direction, so as to realize the smooth paving of asphalt materials. If half-width paving construction technology is adopted, a certain gap should be left at the longitudinal joint during paving, and rolling is not allowed. Generally speaking, overlap paving is used by elevation datum. And when rolling the joint position, it is necessary to use the thermal connection method to eliminate the weld traces. If the road structure is a complex multi-layer structure, the longitudinal joints between the upper and lower layers need to be staggered reasonably. The horizontal joint adopts the flat joint construction technology. Determine the position where the support and the paving layer interact with each other as the joint position, and use a slitting machine for joint treatment. Finally, clean up and deal with impurities in the joints in time. When using viscous asphalt for bonding treatment, the paving work is assisted by the screed.

\subsection{Temperature control of the road construction}

When using asphalt pavement, the air, temperature, and humidity of the construction site should be monitored to ensure smooth paving of materials. Then the use of paving equipment should be paid attention to. Choosing the right machinery is very important. Generally, crawler paver is used, which has good high temperature resistance and a positive effect on the construction of road and 
bridge asphalt mixture. In addition, a variety of equipment can be used for construction, which improves construction efficiency, shortens construction period and saves construction cost. And it is important to keep a certain distance during construction.

\subsection{Reasonable use of maintenance machinery and equipment for asphalt road}

In the current maintenance and management of road and bridge projects in China, the main advantage of management lies in its high efficiency and high quality. At the same time, it is necessary to have good operating skills and levels so as not to affect the environment. Therefore, relevant road and bridge management departments should increase the service life of roads and bridges by strengthening the introduction and innovation of mechanical equipment. Foreign advanced technology can be learned to improve equipment efficiency by improving equipment automation, intelligence and energy saving. Road and bridge engineering maintenance ensures the development of mechanization, improves maintenance functions and related technologies, and improves the safety and stability of the use of mechanical equipment, so as to promote the smooth completion of maintenance work and improve the efficiency of the use of the road.

\subsection{Slurry seal technology}

If cracks are found on the asphalt road in time, and the cracks occur at a relatively early stage, they can be treated with mud sealing technology (see Figure 2). This technology mainly uses emulsified asphalt slurry to seal to enhance the slip resistance and wear resistance of the asphalt road seal itself, but it cannot completely control the expansion of cracks.

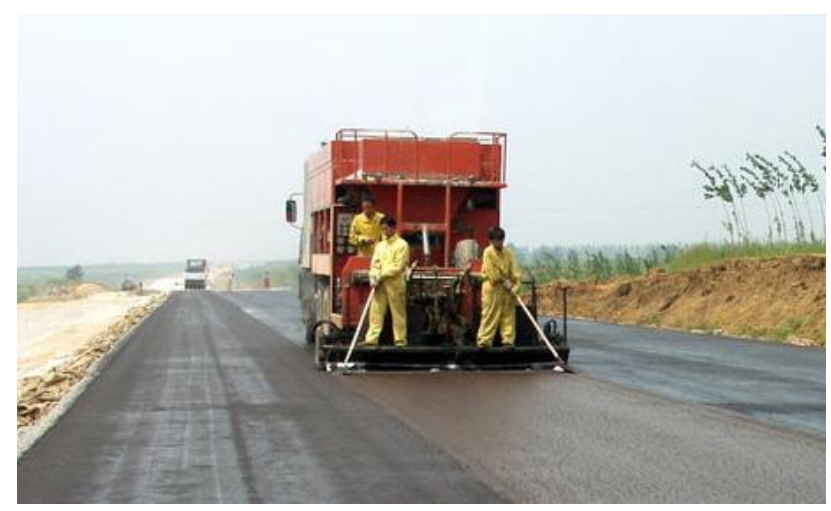

Figure 2. Slurry seal technology

\subsection{Control and management of construction materials}

In the process of road and bridge construction, the use of asphalt mixture construction technology to strictly control the quality of construction materials is the most basic step to ensure construction quality. In this process, it is first necessary to select the most cost-effective construction materials according to the project requirements and the characteristics of the construction materials, and to control the quality of the materials from the source of the material manufacturer. Secondly, the quality must be strictly controlled when transporting materials, and the materials should not be stacked randomly when transported to the construction site, especially some special materials, such as cement or steel bars, which should be stored in a centralized manner. For materials with high storage environment requirements, serious quality problems may occur if they are not properly stored. Therefore, it is necessary to pay attention to the storage environment and let professionals handle them. Finally, in the actual construction process, it is necessary to strictly control the cutting quality of the steel bars, and a cutting machine or a grinding wheel can be used, so as to avoid losses caused by the arc during the cutting process.

\subsection{Choice of advanced maintenance technology}

In the maintenance and management of road and bridge projects, maintenance must be in an important position. Advanced maintenance management technology can change the concept of existing maintenance work, improve the level of rural road maintenance work, and prevent the occurrence of engineering safety accidents. As a management department, it is necessary to formulate maintenance techniques in advance and take appropriate remedial measures when unexpected situations occur. In particular, the congestion and disease problems of roads and bridges need to be solved, and the maintenance level must be effectively improved to ensure the service life of roads and bridges. In addition, it is necessary to use new energy and new materials as much as possible to achieve energy saving in the road maintenance. At the same time, attention should also be paid to drainage problems, because water erosion will seriously damage the road surface, and timely measures and maintenance techniques must be taken to eliminate diseases and other problems, thereby increasing the service life of the road.

\section{ConClusion}

In summary, there are many factors that affect the appearance of cracks on roads and bridges. The types of cracks caused by different factors may be the same or different, so a high degree of attention must be paid. In order to achieve this goal, it is necessary to deal with different types of cracks to improve the performance of roads and bridges.

\section{References}

1. Qun Tuo. Construction and treatment technology for cracks in asphalt pavement of roads and bridges $[\mathrm{J}]$. Green Building Materials, 2019(06): 114+116.

2. Lingsen $\mathrm{Xu}$. Research on construction treatment technology of asphalt pavement cracks in roads and bridges $[\mathrm{J}]$. Construction Technology Development, 2019, 46(04): 120-121.

3. QifengLi, Huirong Zhang. Construction and treatment technology of asphalt pavement cracks in roads and bridges[J]. World of Transportation, 2018(28): 88-89.

4. Ruixiao Sun. Analysis of construction and treatment technology for cracks in asphalt pavement of roads and bridges $[\mathrm{J}]$. Modern Property (mid-day issue), 2018(06): 182 
5. Aihua Wang. Construction and treatment technology for cracks in asphalt pavement of roads and bridges[J]. Doors and Windows, 2018(03): 113. 